\title{
Nested sampling cross-checks using order statistics
}

\author{
Andrew Fowlie ${ }^{1 \star}$, Will Handley ${ }^{2,3} \dagger$, and Liangliang $\mathrm{Su}^{1} \ddagger$ \\ ${ }^{1}$ Department of Physics and Institute of Theoretical Physics, Nanjing Normal University, Nanjing, Jiangsu 210023, China \\ ${ }^{2}$ Astrophysics Group, Cavendish Laboratory, J.J.Thomson Avenue, Cambridge, CB3 OHE, UK \\ ${ }^{3}$ Kavli Institute for Cosmology, Madingley Road, Cambridge, CBз OHA, UK
}

\begin{abstract}
Nested sampling (NS) is an invaluable tool in data analysis in modern astrophysics, cosmology, gravitational wave astronomy and particle physics. We identify a previously unused property of NS related to order statistics: the insertion indexes of new live points into the existing live points should be uniformly distributed. This observation enabled us to create a novel cross-check of single NS runs. The tests can detect when an NS run failed to sample new live points from the constrained prior and plateaus in the likelihood function, which break an assumption of NS and thus leads to unreliable results. We applied our cross-check to NS runs on toy functions with known analytic results in $2-50$ dimensions, showing that our approach can detect problematic runs on a variety of likelihoods, settings and dimensions. As an example of a realistic application, we cross-checked NS runs performed in the context of cosmological model selection. Since the cross-check is simple, we recommend that it become a mandatory test for every applicable NS run.
\end{abstract}

Key words: methods: statistical - methods: data analysis - methods: numerical

\section{INTRODUCTION}

Nested sampling (NS) was introduced by Skilling in 2004 (Skilling 2004; Skilling 2006) as a novel algorithm for computing Bayesian evidences and posterior distributions. The algorithm requires few tuning parameters and can cope with traditionally-challenging multimodal and degenerate functions. As a result, popular implementations such as MultiNest (Feroz \& Hobson 2008; Feroz et al. 2009, 2013), PolyChord (Handley et al. 2015a; Handley et al. 2015b) and dynesty (Speagle 2020) have become invaluable tools in modern cosmology (Mukherjee et al. 2006; Easther \& Peiris 2012; Martin et al. 2014; Hlozek et al. 2015; Audren et al. 2013; Planck Collaboration et al. 2018), astrophysics (Trotta et al. 2011; Liddle 2007; Buchner et al. 2014), gravitational wave astronomy (Veitch et al. 2015; Abbott et al. 2016b,a; Ashton et al. 2019), and particle physics (Trotta et al. 2008; Feroz et al. 2008; Buchmueller et al. 2014; Martinez et al. 2017). Other NS applications include statistical physics (Bolhuis \& Csányi 2018; Martiniani et al. 2014; PÃartay et al. 2010; Pártay et al. 2014; Baldock et al. 2017; Nielsen 2013), condensed matter physics (Baldock et al. 2016), and biology (Russel et al. 2018; Johnson et al. 2014).

In this work, we propose a cross-check of an important assumption in NS that works on single NS runs. This im-

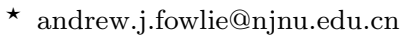

$\dagger$ wh260@cam.ac.uk

† 191002001@stu.njnu.edu.cn proves upon previous tests of NS that required toy functions with known analytic properties (Buchner 2016) or multiple runs (Higson et al. 2019). The cross-check detects faults in the compression of the parameter space that lead to biased estimates of the evidence. We demonstrate our method on toy functions and previous NS runs used for model selection in cosmology (Handley 2019b). We anticipate that the cross-check could be applied as broadly as NS itself.

The paper is structured as follows. After recapitulating the relevant aspects of NS in section 2, we introduce our approach in section 3 . We apply our methods to toy functions and a cosmological likelihood in section 4. We briefly discuss the possibility of using the insertion indexes to debias NS evidence estimates in section 5 before concluding in section 6 .

\section{NS ALGORITHM}

To establish our notation and explain our cross-check, we briefly summarize the NS algorithm. For more detailed and pedagogical introductions, see e.g., (Skilling 2006; Feroz et al. 2009; Handley et al. 2015a; Speagle 2020). NS is primarily an algorithm for computing the Bayesian evidence of a model in light of data. Consider a model with parameters $\boldsymbol{\Theta}$. The evidence may be written

$\mathcal{Z} \equiv \int_{\Omega_{\Theta}} \mathcal{L}(\Theta) \pi(\Theta) \mathrm{d} \Theta$, 
where $\pi(\boldsymbol{\Theta})$ is a prior density for the parameters and $\mathcal{L}(\boldsymbol{\Theta})$ is a likelihood function describing the probability of the observed experimental data. The evidence is a critical ingredient in Bayesian model selection in which models are compared by Bayes factors, since Bayes factors are ratios of evidences for two models,

$B_{10} \equiv \frac{\mathcal{Z}_{1}}{\mathcal{Z}_{0}}$.

The Bayes factor $B_{10}$ tells us hows much more we should believe in model 1 relative to model 0 in light of experimental data. For an introduction to Bayes factors, see e.g., (Kass \& Raftery 1995).

NS works by casting eq. (1) as a one-dimensional integral via the volume variable,

$X(\lambda)=\int_{\mathcal{L}(\boldsymbol{\Theta})>\lambda} \pi(\boldsymbol{\Theta}) \mathrm{d} \boldsymbol{\Theta}$.

This is the prior volume enclosed within the iso-likelihood contour defined by $\lambda$. The evidence may then be written as

$\mathcal{Z}=\int_{0}^{1} \mathcal{L}(X) \mathrm{d} X$

where in the overloaded notation $\mathcal{L}(X)$ is the inverse of $X(\lambda)$.

The remaining challenge is computing the onedimensional integral in eq. (4). In NS we begin from $n_{\text {live }}$ live points drawn from the prior. At each iteration of the NS algorithm, we discard the point with the smallest likelihood, $\mathcal{L}^{\star}$, and sample a replacement drawn from the constrained prior, that is, drawn from $\pi(\boldsymbol{\Theta})$ subject to $\mathcal{L}(\boldsymbol{\Theta})>\mathcal{L}^{\star}$. By the statistical properties of random samples drawn from the constrained prior, we expect that the volume $X\left(\mathcal{L}^{\star}\right)$ compresses by $t$ at each iteration, where

$\langle\log t\rangle=-\frac{1}{n_{\text {live }}}$.

This enables us to estimate the volume at the $i$-th iteration by $X_{i} \equiv X\left(\mathcal{L}_{i}^{\star}\right)=e^{-i / n_{\text {live }}}$ and write the one-dimensional integral using the trapezium rule,

$\mathcal{Z} \approx \sum_{i} \mathcal{L}_{i}^{\star} w_{i}, \quad w_{i}=\frac{1}{2}\left(X_{i-1}-X_{i+1}\right)$.

The algorithm terminates once an estimate of the maximum remaining evidence, $\Delta \mathcal{Z}$, is less than a specified fraction, $\epsilon$, of the total evidence found,

$$
\frac{\Delta \mathcal{Z}}{\mathcal{Z}}<\epsilon
$$

The main numerical problem in an implementation of NS is efficiently sampling from the constrained prior.

\subsection{Sampling from the constrained prior}

Because rejection sampling from the entire prior would be impractically slow as the volume compresses exponentially, implementations of NS typically employ specialised subalgorithms to sample from the constrained prior. When these subalgorithms fail, the evidences may be unreliable. This was considered the most severe drawback of the NS algorithm in (Salomone et al. 2018).

One such subalgorithm is ellipsoidal sampling (Mukherjee et al. 2006; Feroz \& Hobson 2008), a rejection sampling algorithm in which the live points are bounded by a set of ellipsoids. Potential live points are sampled from the ellipsoids and accepted only if $\mathcal{L}>\mathcal{L}^{\star}$. Ellipsoidal NS is implemented in MultiNest (Feroz \& Hobson 2008; Feroz et al. 2009, 2013). For this to faithfully sample from the constrained prior, the ellipsoids must completely enclose the iso-likelihood contour defined by $\mathcal{L}^{\star}$. To ensure this is the case, the ellipsoids are expanded by a factor $1 /$ efr, with efr $=0.3$ recommended for reliable evidences.

Slice sampling (Neal 2003) is an alternative scheme for sampling from the constrained prior (Aitken \& Akman 2013; Handley et al. 2015a). A chord is drawn from a live point across the entire region enclosed by the iso-likelihood contour and a candidate point is drawn uniformly from along the chord. This is repeated $n_{r}$ times to reduce correlations between the new point and the original live point. Slice sampling is implemented in PolyChord (Handley et al. 2015a; Handley et al. 2015b). The recommend number of repeats is $n_{r}=2 d$ for a $d$-dimensional function.

\subsection{Plateaus in the likelihood}

Plateaus in the likelihood function, i.e., regions in which $\mathcal{L}(\boldsymbol{\Theta})=$ const., were discussed in (Skilling 2004; Skilling 2006) and more recently in (Schittenhelm \& Wacker 2020). In (Schittenhelm \& Wacker 2020) it was stressed that they can lead to faulty estimates of the compression. In such cases, the live points are not uniformly distributed in $X$ (eq. (3)), violating assumptions in eq. (5).

\section{USING INSERTION INDEXES}

By insertion index, we mean the index at which an element must be inserted to maintain order in an sorted list. With a left-sided convention, the insertion index $i$ of a sample $y$ in an sorted list $o$ is such that

$o_{i-1}<y \leq o_{i}$.

The key idea in this paper is to use the insertion indexes of new live points relative to existing live points sorted by enclosed prior volume, $X$, to detect problems in sampling from the constrained prior. Since the relationship between volume and likelihood is monotonic, we can sort by volume by sorting by likelihood. If new live points are genuinely sampled from the constrained prior leading to a uniform distribution in $X$, the insertion indexes, $i$, should be discrete uniformly distributed from 0 to $n_{\text {live }}-1$,

$i \sim \mathcal{U}\left(0, n_{\text {live }}-1\right)$.

This result from order statistics is proven in appendix A. During a NS run of $n_{\text {iter }}$ iterations we thus find $n_{\text {iter }}$ insertion indexes that should be uniformly distributed. Imagine, however, that during a NS run using ellipsoidal sampling, the ellipsoids encroached on the true iso-likelihood contour. In that case, the insertion indexes near the lowest-likelihood live points could be disfavoured, and the distribution of insertion indexes would deviate from uniformity. Alternatively, imagine that the likelihood function contains a plateau. Any initial live points that lie in the plateau share the same insertion index, leading to many repeated indexes and a strong deviation from a uniform distribution. 


\begin{tabular}{|c|c|c|c|c|c|c|c|c|c|}
\hline efr & $d$ & Analytic $\log \mathcal{Z}$ & Mean $\log \mathcal{Z} \pm \Delta \log \mathcal{Z}$ & $\sigma_{\log Z}$ & SEM $\log \mathcal{Z}$ & Inaccuracy & Bias & Median $p$-value & Median rolling $p$-value \\
\hline \multicolumn{10}{|c|}{ Gaussian } \\
\hline 0.10 & 2 & 0 & $-0.00 \pm 0.10$ & 0.10 & 0.01 & -0.04 & -0.47 & 0.50 & 0.49 \\
\hline 0.10 & 10 & 0 & $0.01 \pm 0.23$ & 0.21 & 0.02 & 0.04 & 0.48 & 0.59 & 0.60 \\
\hline 0.10 & 30 & 0 & $0.38 \pm 0.41$ & 0.36 & 0.04 & 0.93 & 10.56 & 0.52 & $2.7 \cdot 10^{-4}$ \\
\hline 0.10 & 50 & 0 & $2.08 \pm 0.52$ & 0.50 & 0.05 & 3.98 & 41.25 & 0.38 & $4.5 \cdot 10^{-24}$ \\
\hline 1 & 2 & 0 & $-0.00 \pm 0.10$ & 0.10 & 0.01 & -0.04 & -0.46 & 0.52 & 0.49 \\
\hline 1 & 10 & 0 & $0.57 \pm 0.23$ & 0.22 & 0.02 & 2.43 & 26.07 & 0.21 & $1.2 \cdot 10^{-4}$ \\
\hline 1 & 30 & 0 & $2.35 \pm 0.40$ & 0.37 & 0.04 & 5.83 & 63.82 & 0.23 & $2.2 \cdot 10^{-23}$ \\
\hline 1 & 50 & 0 & $4.06 \pm 0.52$ & 0.44 & 0.04 & 7.81 & 92.99 & 0.30 & $1.3 \cdot 10^{-34}$ \\
\hline 10 & 2 & 0 & $-64.75 \pm 0.11$ & 93.15 & 9.31 & -532.44 & -6.95 & $7.7 \cdot 10^{-3}$ & 0.06 \\
\hline 10 & 10 & 0 & $2.81 \pm 0.23$ & 0.19 & 0.02 & 12.30 & 150.55 & $2.1 \cdot 10^{-6}$ & $1.7 \cdot 10^{-19}$ \\
\hline 10 & 30 & 0 & $4.30 \pm 0.40$ & 0.25 & 0.02 & 10.75 & 174.47 & 0.02 & $3.1 \cdot 10^{-68}$ \\
\hline 10 & 50 & 0 & $6.04 \pm 0.52$ & 0.31 & 0.03 & 11.66 & 197.79 & 0.08 & $1.1 \cdot 10^{-93}$ \\
\hline \multicolumn{10}{|c|}{ Rosenbrock } \\
\hline 0.10 & 2 & -5.80 & $-5.79 \pm 0.07$ & 0.07 & 0.01 & 0.22 & 2.32 & 0.50 & 0.54 \\
\hline 1 & 2 & -5.80 & $-5.72 \pm 0.07$ & 0.05 & 0.01 & 1.18 & 15.31 & 0.08 & 0.32 \\
\hline 10 & 2 & -5.80 & $-5.77 \pm 0.07$ & 0.34 & 0.03 & 0.65 & 1.09 & $9.6 \cdot 10^{-3}$ & 0.07 \\
\hline \multicolumn{10}{|l|}{ Shells } \\
\hline 0.10 & 2 & -1.75 & $-1.75 \pm 0.05$ & 0.05 & 0.01 & -0.06 & -0.64 & 0.55 & 0.55 \\
\hline 0.10 & 10 & -14.59 & $-14.59 \pm 0.12$ & 0.13 & 0.01 & 0.02 & 0.16 & 0.57 & 0.56 \\
\hline 0.10 & 30 & -60.13 & $-59.61 \pm 0.24$ & 0.21 & 0.02 & 2.11 & 24.29 & 0.37 & $7.3 \cdot 10^{-6}$ \\
\hline 0.10 & 50 & -112.42 & $-110.15 \pm 0.33$ & 0.20 & 0.02 & 6.87 & 115.58 & 0.07 & $3.7 \cdot 10^{-23}$ \\
\hline 1 & 2 & -1.75 & $-1.71 \pm 0.05$ & 0.05 & 0.00 & 0.79 & 8.52 & $4.7 \cdot 10^{-3}$ & 0.10 \\
\hline 1 & 10 & -14.59 & $-13.92 \pm 0.12$ & 0.10 & 0.01 & 5.57 & 65.88 & 0.02 & $1.1 \cdot 10^{-5}$ \\
\hline 1 & 30 & -60.13 & $-57.57 \pm 0.24$ & 0.17 & 0.02 & 10.67 & 151.79 & $7.7 \cdot 10^{-3}$ & $1.4 \cdot 10^{-20}$ \\
\hline 1 & 50 & -112.42 & $-107.97 \pm 0.33$ & 0.18 & 0.02 & 13.63 & 218.07 & $3.5 \cdot 10^{-3}$ & $3.6 \cdot 10^{-37}$ \\
\hline 10 & 2 & -1.75 & $-1.73 \pm 0.05$ & 0.12 & 0.01 & 0.39 & 1.45 & 0.07 & 0.18 \\
\hline 10 & 10 & -14.59 & $-11.73 \pm 0.11$ & 0.09 & 0.01 & 25.56 & 321.53 & $6.8 \cdot 10^{-18}$ & $1.7 \cdot 10^{-19}$ \\
\hline 10 & 30 & -60.13 & $-55.41 \pm 0.24$ & 0.13 & 0.01 & 20.03 & 367.16 & $3.0 \cdot 10^{-6}$ & $9.3 \cdot 10^{-66}$ \\
\hline 10 & 50 & -112.42 & $-105.82 \pm 0.32$ & 0.13 & 0.01 & 20.42 & 480.50 & $9.3 \cdot 10^{-6}$ & $2.2 \cdot 10^{-92}$ \\
\hline \multicolumn{10}{|c|}{ Mixture } \\
\hline 0.10 & 2 & -8.19 & $-8.18 \pm 0.06$ & 0.06 & 0.01 & 0.08 & 0.68 & 0.47 & 0.58 \\
\hline 0.10 & 10 & -40.94 & $-40.56 \pm 0.16$ & 0.10 & 0.01 & 2.46 & 38.50 & 0.18 & 0.46 \\
\hline 0.10 & 20 & -81.89 & $-79.04 \pm 0.22$ & 0.14 & 0.01 & 13.05 & 210.43 & $2.7 \cdot 10^{-6}$ & $1.5 \cdot 10^{-3}$ \\
\hline 1 & 2 & -8.19 & $-8.16 \pm 0.06$ & 0.06 & 0.01 & 0.55 & 5.65 & 0.34 & 0.58 \\
\hline 1 & 10 & -40.94 & $-38.66 \pm 0.15$ & 0.08 & 0.01 & 15.30 & 304.65 & $1.0 \cdot 10^{-9}$ & $1.7 \cdot 10^{-4}$ \\
\hline 1 & 20 & -81.89 & $-76.83 \pm 0.21$ & 0.13 & 0.01 & 23.69 & 388.70 & $1.4 \cdot 10^{-11}$ & $5.6 \cdot 10^{-7}$ \\
\hline 10 & 2 & -8.19 & $-8.19 \pm 0.06$ & 0.19 & 0.02 & 0.08 & 0.15 & 0.08 & 0.18 \\
\hline 10 & 10 & -40.94 & $-36.74 \pm 0.14$ & 0.07 & 0.01 & 29.36 & 598.27 & $6.1 \cdot 10^{-24}$ & $1.1 \cdot 10^{-8}$ \\
\hline 10 & 20 & -81.89 & $-74.70 \pm 0.21$ & 0.12 & 0.01 & 34.51 & 602.35 & $4.9 \cdot 10^{-17}$ & $7.1 \cdot 10^{-13}$ \\
\hline
\end{tabular}

Table 1. Summary of results of our insertion index cross-check for MultiNest. The numerical results are the average from 100 runs. Biases and inaccuracies greater than 3 and $p$-values less than 0.01 are highlighted by red.

Thus, we can perform a statistical test on the insertion indexes to detect deviations from a uniform distribution. The choice of test isn't important to our general idea of using information in the insertion indexes, though in our examples we use a Kolmogorov-Smirnov (KS) test (Smirnov 1948; Kolmogorov 1933), which we found to be powerful, to compute a $p$-value from all the iterations. We describe the KS test in appendix B.

Excepting plateaus, deviations from uniformity are caused by a change in the distribution of new live points with respect to the existing live points. Since there is no technical challenge in sampling the initial live points from the prior, failures should typically occur during a run and thus be accompanied by a change in the distribution. In runs with many iterations in which a change occurs only once, the power of the test may be diluted by the many iterations before and after the distribution changes, as the insertion indexes before and after the change should be uniformly distributed. To mitigate this, we also perform multiple tests on chunks of iterations, find the smallest resulting $p$-value and apply a correction for multiple testing. We later refer to this as the rolling $p$-value. Since the volume compresses by $e$ in $n_{\text {live }}$ iterations, we pick $n_{\text {live }}$ as a reasonable size for a chunk of iterations. We treat each chunk as independent. The procedure for computing the rolling $p$-value is detailed in algorithm 1. For clarity, let us stress that we later present $p$-values from all the iterations and rolling $p$-values. Functionality to perform these tests on MultiNest and PolyChord output is now included in anesthetic-1.3.6 (Handley 2019c).

We furthermore neglect correlations between the insertion indexes. Finally, we stress that the magnitude of the deviation from uniform, as well as the $p$-value, should be noted. A small $p$-value alone isn't necessarily cause for concern, if the departure from uniformity is negligible. 


\begin{tabular}{|c|c|c|c|c|c|c|c|c|c|}
\hline$d / n_{r}$ & $d$ & Analytic $\log \mathcal{Z}$ & Mean $\log \mathcal{Z} \pm \Delta \log \mathcal{Z}$ & $\sigma_{\log Z}$ & SEM $\log \mathcal{Z}$ & Inaccuracy & Bias & Median $p$-value & Median rolling $p$-value \\
\hline \multicolumn{10}{|c|}{ Gaussian } \\
\hline 0.50 & 2 & 0 & $0.01 \pm 0.11$ & 0.11 & 0.01 & 0.11 & 1.03 & 0.54 & 0.60 \\
\hline 0.50 & 10 & 0 & $-0.00 \pm 0.23$ & 0.23 & 0.02 & -0.01 & -0.10 & 0.48 & 0.52 \\
\hline 0.50 & 30 & 0 & $-0.06 \pm 0.41$ & 0.37 & 0.04 & -0.15 & -1.61 & 0.54 & 0.57 \\
\hline 0.50 & 50 & 0 & $-0.05 \pm 0.52$ & 0.59 & 0.06 & -0.10 & -0.85 & 0.58 & 0.51 \\
\hline 1 & 2 & 0 & $-0.02 \pm 0.11$ & 0.10 & 0.01 & -0.19 & -1.96 & 0.42 & 0.48 \\
\hline 1 & 10 & 0 & $-0.04 \pm 0.23$ & 0.18 & 0.02 & -0.17 & -2.20 & 0.55 & 0.59 \\
\hline 1 & 30 & 0 & $-0.83 \pm 0.41$ & 0.40 & 0.04 & -2.06 & -20.73 & 0.61 & 0.46 \\
\hline 1 & 50 & 0 & $-2.48 \pm 0.52$ & 0.46 & 0.05 & -4.73 & -54.22 & 0.49 & 0.59 \\
\hline 2 & 2 & 0 & $-0.01 \pm 0.11$ & 0.15 & 0.02 & -0.12 & -0.89 & 0.47 & 0.53 \\
\hline 10 & 10 & 0 & $2.20 \pm 0.23$ & 0.73 & 0.07 & 9.50 & 30.29 & 0.13 & 0.22 \\
\hline 30 & 30 & 0 & $48.37 \pm 0.64$ & 6.85 & 0.69 & 112.25 & 70.58 & $8.2 \cdot 10^{-10}$ & 0.02 \\
\hline 50 & 50 & 0 & $69.74 \pm 3.05$ & 6.55 & 0.65 & 23.31 & 106.51 & $8.0 \cdot 10^{-86}$ & $1.4 \cdot 10^{-6}$ \\
\hline \multicolumn{10}{|c|}{ Rosenbrock } \\
\hline 0.50 & 2 & -5.80 & $-5.79 \pm 0.07$ & 0.08 & 0.01 & 0.20 & 1.71 & 0.42 & 0.46 \\
\hline 1 & 2 & -5.80 & $-5.81 \pm 0.07$ & 0.08 & 0.01 & -0.05 & -0.55 & 0.44 & 0.52 \\
\hline 2 & 2 & -5.80 & $-5.83 \pm 0.07$ & 0.09 & 0.01 & -0.36 & -2.82 & 0.56 & 0.49 \\
\hline \multicolumn{10}{|l|}{ Shells } \\
\hline 0.50 & 2 & -1.75 & $-1.74 \pm 0.05$ & 0.05 & 0.00 & 0.16 & 1.54 & 0.13 & 0.13 \\
\hline 0.50 & 10 & -14.59 & $-14.59 \pm 0.12$ & 0.12 & 0.01 & 0.02 & 0.12 & 0.50 & 0.48 \\
\hline 0.50 & 30 & -60.13 & $-60.12 \pm 0.25$ & 0.24 & 0.02 & 0.03 & 0.29 & 0.56 & 0.55 \\
\hline 0.50 & 50 & -112.42 & $-112.33 \pm 0.34$ & 0.33 & 0.03 & 0.27 & 2.65 & 0.40 & 0.58 \\
\hline 1 & 2 & -1.75 & $-1.75 \pm 0.05$ & 0.04 & 0.00 & -0.02 & -0.30 & 0.01 & 0.01 \\
\hline 1 & 10 & -14.59 & $-14.59 \pm 0.12$ & 0.12 & 0.01 & 0.02 & 0.19 & 0.49 & 0.61 \\
\hline 1 & 30 & -60.13 & $-60.46 \pm 0.25$ & 0.23 & 0.02 & -1.36 & -14.57 & 0.48 & 0.53 \\
\hline 1 & 50 & -112.42 & $-113.52 \pm 0.34$ & 0.32 & 0.03 & -3.26 & -34.47 & 0.50 & 0.51 \\
\hline 2 & 2 & -1.75 & $-1.74 \pm 0.05$ & 0.06 & 0.01 & 0.06 & 0.43 & $6.1 \cdot 10^{-6}$ & $2.1 \cdot 10^{-5}$ \\
\hline 10 & 10 & -14.59 & $-14.05 \pm 0.12$ & 0.36 & 0.04 & 4.42 & 15.01 & 0.09 & 0.09 \\
\hline 30 & 30 & -60.13 & $-38.78 \pm 0.21$ & 1.34 & 0.13 & 103.26 & 159.47 & $3.5 \cdot 10^{-5}$ & $5.2 \cdot 10^{-3}$ \\
\hline 50 & 50 & -112.42 & $-64.20 \pm 0.63$ & 5.17 & 0.52 & 103.63 & 93.31 & $5.2 \cdot 10^{-12}$ & $3.8 \cdot 10^{-7}$ \\
\hline \multicolumn{10}{|c|}{ Mixture } \\
\hline 0.50 & 2 & -8.19 & $-8.17 \pm 0.06$ & 0.06 & 0.01 & 0.35 & 3.33 & 0.45 & 0.56 \\
\hline 0.50 & 10 & -40.94 & $-40.87 \pm 0.16$ & 0.15 & 0.02 & 0.49 & 5.12 & 0.43 & 0.52 \\
\hline 0.50 & 20 & -81.89 & $-81.75 \pm 0.23$ & 0.24 & 0.02 & 0.61 & 5.70 & 0.51 & 0.51 \\
\hline 1 & 2 & -8.19 & $-8.16 \pm 0.06$ & 0.06 & 0.01 & 0.39 & 3.78 & 0.44 & 0.49 \\
\hline 1 & 10 & -40.94 & $-40.85 \pm 0.16$ & 0.16 & 0.02 & 0.59 & 5.71 & 0.48 & 0.45 \\
\hline 1 & 20 & -81.89 & $-81.72 \pm 0.22$ & 0.27 & 0.03 & 0.73 & 6.18 & 0.52 & 0.59 \\
\hline 2 & 2 & -8.19 & $-8.18 \pm 0.06$ & 0.09 & 0.01 & 0.09 & 0.58 & 0.52 & 0.49 \\
\hline 10 & 10 & -40.94 & $-40.90 \pm 0.18$ & 1.31 & 0.13 & 0.77 & 0.31 & $7.2 \cdot 10^{-3}$ & 0.27 \\
\hline 20 & 20 & -81.89 & $-88.28 \pm 0.64$ & 5.80 & 0.58 & -11.25 & -11.03 & $4.5 \cdot 10^{-15}$ & 0.01 \\
\hline
\end{tabular}

Table 2. Summary of results of our insertion index cross-check for PolyChord. See table 1 for further details. In this table we show $d / n_{r}$, which may be thought of as a "PolyChord efficiency" analogue of the MultiNest efficiency efr.

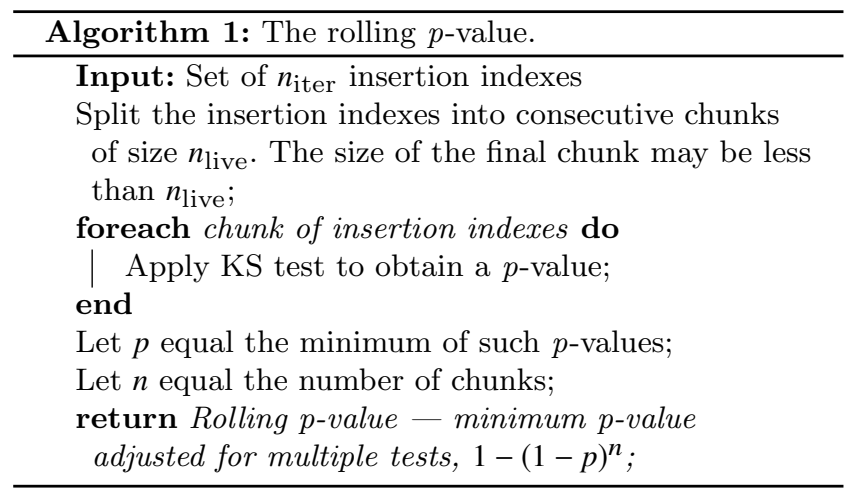

\section{EXAMPLES}

\subsection{Toy functions}

We now present detailed numerical examples of our crosscheck using NS runs on toy functions using MultiNest3.12 (Feroz \& Hobson 2008; Feroz et al. 2009, 2013) and Poly-
Chord-1.17.1 (Handley et al. 2015a; Handley et al. 2015b). We chose toy functions with known analytic evidences or precisely known numerical estimates of the evidence to demonstrate that biased results from NS are detectable with our approach. The toy functions are described in appendix C.

We performed 100 MultiNest and PolyChord runs on each toy function to study the statistical properties of their outputs. We used $n_{\text {live }}=1000$ and $\epsilon=0.01$ throughout. To generate biased NS runs, we used inappropriate settings, e.g., efr $>1$ in MultiNest or few repeats $n_{r}<d$ in slice sampling in PolyChord, and difficult toy functions with $d \geq 30$. We post-processed the results using anesthetic (Handley 2019d).

We summarise our results by the average $\log \mathcal{Z}$ and error estimate $\Delta \log \mathcal{Z}$, and by the median $p$-value from all the insertion indexes and the median running $p$-value. We furthermore report the standard error on the mean, SEM $\log \mathcal{Z}$, and the standard deviation, $\sigma_{\log \mathcal{Z}}$. We use the error 
estimates to compute the average inaccuracy and bias,

$$
\begin{aligned}
\text { inaccuracy } & =\frac{\log \mathcal{Z}-\text { analytic }}{\Delta \log \mathcal{Z}}, \\
\text { bias } & =\frac{\log \mathcal{Z}-\text { analytic }}{\text { SEM } \log \mathcal{Z}} .
\end{aligned}
$$

The inaccuracy shows whether the uncertainty reported by a code from single runs was reasonable.

We present our numerical results using MultiNest and PolyChord in tables 1 and 2, respectively. First, for the Gaussian function, the MultiNest estimates of $\log \mathcal{Z}$ were significantly biased for $d=30$ and 50 for all efr settings, and for $d=2$ and 10 for efr $=10$. Our cross-check was successful, as the $p$-values corresponding to the biased results were tiny.

For the Rosenbrock function, our cross-check detected a problem with MultiNest runs with $d=2$ and efr $=10$, even though the MultiNest evidence estimate was not biased. It did not detect a problem with efr $=1$, even though the $\log \mathcal{Z}$ estimate was biased. This was, however, the only problem for which this occurred for MultiNest.

For the shells function, the MultiNest estimates of $\log \mathcal{Z}$ were biased for many combinations of $d$ and efr. The biased results were all identified by our cross-check with tiny $p$-values. Indeed, when $d=50$, even with efr $=0.1$, we saw a bias of about 115 and a median rolling $p$-value of about $10^{-23}$.

Lastly, the $d=20$ mixture functions are particularly important, as MultiNest was known to produce biased results even with efr $=0.1$. Using all the insertion indexes, we find $p$-value $\approx 10^{-6}$ for this function, i.e., our cross-check successfully detects these failures.

In the analogous results for PolyChord in table 2 we see fewer significantly biased estimates throughout, and only three biased results when using the recommended $n_{r}=2 d$ setting, which all occurred in the mixture function. We note, though, that the error estimates from PolyChord were reasonable even in these cases. The most extremely biased results were detected by our cross-check in the Gaussian, shells and mixture functions.

Our cross-check detected faults in the $d=2$ shells function for $n_{r}=1$, despite no evidence of bias in PolyChord results. Perhaps this should not be surprising, as (Salomone et al. 2018; Speagle 2020) suggest that independent samples from the constrained prior are not strictly necessary for correct evidence estimates. The $p$-values, however, increased monotonically as $n_{r}$ was increased, as expected. Lastly, we note that in many more cases than for MultiNest biases were not detected by our cross-check; this may be because the biases are smaller than they were for MultiNest.

In summary, for both MultiNest and PolyChord, we find that our cross-check can detect problematic NS runs in a variety of functions, settings and dimensions, although there is room for refinement. The problem detected by our crosscheck usually leads to a faulty estimate of the evidence, though in a few cases the evidence estimate remains reasonable despite the apparent failure to sample correctly from the constrained prior.

\subsection{Cosmological model selection}

In (Handley 2019b), Handley considered the Bayesian evidence for a spatially closed Universe. Bayesian evidences

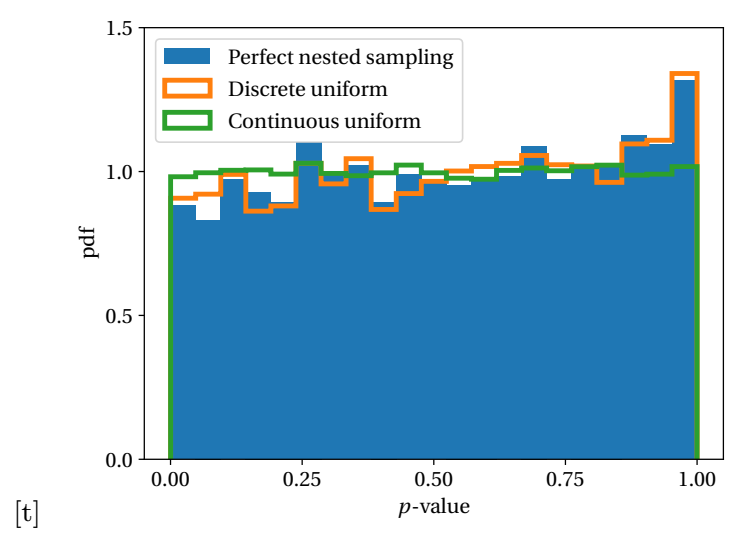

Figure 1. Histogram of $p$-values from tests uniformity of insertion indexes from perfect NS (blue), samples from a discrete uniform distribution (orange) and samples from a continuous uniform distribution (green).

from combinations of four datasets were computed using PolyChord for a spatially flat Universe and a curved Universe. The resulting Bayes factors showed that a closed Universe was favoured by odds of about $50 / 1$ for a particular set of data. There were 22 NS computations in total. The PolyChord results are publicly archived at (Handley 2019a). We ran our cross-check on each of the $22 \mathrm{NS}$ runs in the archived data, finding $p$-values in the range $4 \%$ to $98 \%$. The results do not suggest problems with the NS runs. The $p$-value of $4 \%$ is not particularly alarming, especially considering that we conducted 22 tests. The full results are shown in table 3 .

\subsection{Plateaus}

Let us consider the one-dimensional function in example 2 from (Schittenhelm \& Wacker 2020). The likelihood function is defined piece-wise to be a Gaussian at the center and zero in the tails;

$$
\mathcal{L}(x) \propto \begin{cases}e^{-\frac{(x-\mu)^{2}}{2 \sigma^{2}}} & \left(\frac{x-\mu}{\sigma}\right)^{2} \leq 1 \\ 0 & \text { elsewhere. }\end{cases}
$$

for $\mu=\frac{1}{2}$ and $\sigma=1$. The prior is uniform from -3 to 3 . We confirm that the NS algorithm produces biased estimates of the evidence in this function. However, since the likelihood is zero in $5 / 6$ of the prior, approximately $5 / 6$ of the initial live points have a likelihood of zero and share the same insertion index from eq. (8). This results in a tiny $p$-value $\simeq 0$ in our test.

\subsection{Perfect NS}

Lastly, we simulated perfect NS runs that correctly sample from the constrained prior. We simulated them by directly sampling compression factors from uniform distributions and never computing any likelihoods. Of course, with no likelihood we cannot compute an evidence, but we can simulate insertion indexes. We performed 10,000 runs of perfect NS with 10,000 iterations and computed the $p$-value via our KS test.

We furthermore computed 100,000 $p$-values from a KS 


\begin{tabular}{lcccc} 
& \multicolumn{2}{c}{ Flat } & \multicolumn{2}{c}{ Curved } \\
\cline { 2 - 5 } Data & $p$-value & Rolling $p$-value & $p$-value & Rolling $p$-value \\
\hline BAO & 0.89 & 0.82 & 0.07 & 0.05 \\
lensing+BAO & 0.72 & 0.54 & 0.19 & 0.43 \\
lensing & 0.26 & 0.14 & 0.04 & 0.64 \\
lensing+SH ES & 0.08 & 0.08 & 0.78 & 0.04 \\
Planck+BAO & 0.39 & 0.56 & 0.14 & 0.43 \\
Planck+lensing+BAO & 0.68 & 0.69 & 0.70 & 0.27 \\
Planck+lensing & 0.94 & 0.49 & 0.89 & 0.72 \\
Planck+lensing+SH$H_{0} \mathrm{ES}$ & 0.92 & 0.92 & 0.33 & 0.82 \\
Planck & 0.81 & 0.69 & 0.84 & 0.88 \\
Planck+SHES & 0.20 & 0.48 & 0.92 & 0.97 \\
$\mathrm{SH}_{0} \mathrm{ES}$ & 0.59 & 0.59 & 0.98 & 0.98
\end{tabular}

Table 3. Insertion index cross-check applied to NS results from cosmological model selection in (Handley 2019b). We show $p$-values and rolling $p$-values for the NS evidence calculations for flat and curved Universe models with 11 datasets. See (Handley 2019b) for further description of the datasets and models.

test on 10,000 samples drawn from a continuous uniform distribution and on 10,000 samples drawn from a discrete uniform distribution with 1000 bins. We histogram all $p$-values in fig. 1. Of course, the KS $p$-values should be uniformly distributed in the continuous case and it appears that it is (green). The impact of discretization on the KS test is visible (orange) but small with 1000 live points. The further impact of correlations amongst the samples in perfect NS (blue) isn't obvious. A two-sample Kolmogorov-Smirnoff test similar to the one-sample test in appendix B uncovered a slight difference between $p$-values from perfect NS and from a discrete uniform $(p$-value $=0.03)$. This suggests that although the correlations and discretization impact the KS test, the effect is small.

\section{FUTURE USE OF INSERTION INDEXES}

For the purposes of evidence estimation, a nested sampling run is fully encoded by recording the birth contour and death contour of each point (Higson et al. 2018). For the purposes of estimating volume in a statistical way, we generally discard the likelihood information, focussing on the ordering of the contours. This makes sense, as barring the stopping criterion in eq. (7), the underlying nested sampling algorithm is athermal and insensitive to monotonic transformations of the likelihood.

Traditional nested sampling uses the fact that

$P\left(X_{j} \mid X_{j-1}, n_{\text {live }}\right)=\frac{n_{j}}{X_{j-1}}\left(\frac{X_{j}}{X_{j-1}}\right)^{n_{j}-1}\left[0<X_{j}<X_{j-1}\right]$.

In the above, one has essentially marginalised out dependency on everything other than $X_{j-1}$, and compressed the birth-death contour information into a vector encoding the number of live points at each iteration $n_{i}$. One can then use this recursively (alongside the fact that $X_{0}=1$ ) to perform inference on $P(X)$ and therefore the evidence via eqs. (5) and (6).

The critical question therefore is whether this "Skilling compression" from birth-death contours to numbers of live points is lossless or lossy for the purposes of volume estimation (note that it is generically lossy, as it's impossible to go in the reverse direction). The results presented in this paper are suggestive that it is losing some useful information, as insertion indexes do provide further information in the context of a cross check (and are in fact a lossless compression of the birth and death contours). One possibility is that the Skilling compression is lossless in the context of perfect nested sampling, but if a run is biased then you may be able to use insertion indexes to partially correct a biased run. This is the subject of ongoing work by the authors.

\section{CONCLUSIONS}

We identified a previously unknown property of the NS algorithm: the insertion indexes of new live points into the existing live points should be uniformly distributed. This observation enabled us to invent a cross-check of single NS runs. The cross-check can detect when an NS run fails to sample new live points from the constrained prior, which is the most challenging aspect of an efficient implementation of NS, and functions with plateaus in the likelihood function recently identified in (Schittenhelm \& Wacker 2020), both of which can lead to unreliable estimates of the evidence and posterior,

We applied our cross-check to NS runs on several toy functions with known analytic results in $2-50$ dimensions with MultiNest and PolyChord, which sample from the constrained prior using ellipsoidal rejection sampling and slice sampling, respectively. Our numerical results are some of the most detailed checks of MultiNest and PolyChord. We found that our cross-check could detect problematic runs for both codes. Since the idea is relatively simple, we suggest that a cross-check of this kind should become a mandatory test of any NS run. The exact form of the cross-check, however, could be refined. We chose a KS test using all the iterations or the most significant $n_{\text {live }}$ iterations; both choices could be improved. As an example of a realistic application, we furthermore applied our cross-check to results from $22 \mathrm{NS}$ runs performed in the context of cosmological model selection.

Lastly, we speculated that the information contained in the insertion indexes could be used to debias single NS runs or lead to an improved formula for the evidence summation. We outlined a few difficulties and hope our observations lead to further developments.

Future work will involve extending the method to work in the context of a variable number of live points, as well as exploring the larger possibilities of using order statistics to improve NS accuracy and potentially debias runs. 


\section{ACKNOWLEDGMENTS}

The authors would like to thank Gregory Martinez for valuable discussions. We thank the organisers of the GAMBIT XI workshop where some of this work was planned and completed. AF was supported by an NSFC Research Fund for International Young Scientists grant 11950410509. WH was supported by a George Southgate visiting fellowship grant from the University of Adelaide, and STFC IPS grant number G102229.

\section{DATA AVAILABILITY}

The raw data from our 100 runs for tables 1 and 2 are publicly available at (Fowlie et al. 2020). All other data will be shared on reasonable request to the corresponding author.

\section{REFERENCES}

Abbott B. P., et al., 2016a, Phys. Rev., X6, 041015

Abbott B. P., et al., 2016b, Phys. Rev. Lett., 116, 221101

Aitken S., Akman O. E., 2013, BMC Systems Biology, 7, 72

Arnold T. B., Emerson J. W., 2011, The R Journal, 3, 34

Ashton G., et al., 2019, Astrophys. J. Suppl., 241, 27

Audren B., Lesgourgues J., Benabed K., Prunet S., 2013, JCAP, 2013, 001

Baldock R. J. N., Pártay L. B., Bartók A. P., Payne M. C., Csányi G., 2016, Phys. Rev. B, 93, 174108

Baldock R. J. N., Bernstein N., Salerno K. M., Pártay L. B., Csányi G., 2017, Phys. Rev. E, 96, 043311

Beaujean F., Caldwell A., 2013, arXiv e-prints, p. arXiv:1304.7808

Bolhuis P. G., Csányi G., 2018, Phys. Rev. Lett., 120, 250601

Buchmueller O., et al., 2014, Eur. Phys. J. C, 74, 2922

Buchner J., 2016, Statistics and Computing, 26, 383

Buchner J., et al., 2014, Astron. Astrophys., 564, A125

Easther R., Peiris H. V., 2012, Phys. Rev. D, 85, 103533

Feroz F., Hobson M. P., 2008, Mon. Not. Roy. Astron. Soc., 384, 449

Feroz F., Allanach B. C., Hobson M., AbdusSalam S. S., Trotta R., Weber A. M., 2008, JHEP, 10, 064

Feroz F., Hobson M. P., Bridges M., 2009, Mon. Not. Roy. Astron. Soc., 398, 1601

Feroz F., Hobson M. P., Cameron E., Pettitt A. N., 2013, The Open Journal of Astrophysics

Fowlie A., Su L., Handley W., 2020, Supplementary data for Nested sampling cross- checks using order statistics, doi:10.5281/zenodo.3958749

Handley W., 2019a, Curvature tension: evidence for a closed universe (supplementary inference products), doi:10.5281/zenodo.3371152

Handley W., 2019b, arXiv e-prints, p. arXiv:1908.09139

Handley W., 2019c, J. Open Source Softw., 4, 1414

Handley W., 2019d, The Journal of Open Source Software, 4, 1414

Handley W. J., Hobson M. P., Lasenby A. N., 2015a, Mon. Not. Roy. Astron. Soc., 450, L61

Handley W. J., Hobson M. P., Lasenby A. N., 2015b, Mon. Not. Roy. Astron. Soc., 453, 4384

Higson E., Handley W., Hobson M., Lasenby A., et al., 2018, Bayesian Analysis, 13, 873

Higson E., Handley W., Hobson M., Lasenby A., 2019, Mon. Not. Roy. Astron. Soc., 483, 2044

Hlozek R., Grin D., Marsh D. J. E., Ferreira P. G., 2015, Phys. Rev. D, 91, 103512

Johnson R., Kirk P., Stumpf M. P. H., 2014, Bioinformatics, 31, 604
Kass R. E., Raftery A. E., 1995, J. Am. Statist. Assoc., 90, 773

Kolmogorov A., 1933, Giornale dellâĂŹ Instuto Italiano degli Attuari, 4,83

Liddle A. R., 2007, MNRAS, 377, L74

Marsaglia G., Tsang W. W., Wang J., 2003, Journal of Statistical Software, Articles, 8, 1

Martin J., Ringeval C., Trotta R., Vennin V., 2014, JCAP, 03, 039

Martinez G. D., McKay J., Farmer B., Scott P., Roebber E., Putze A., Conrad J., 2017, Eur. Phys. J., C77, 761

Martiniani S., Stevenson J. D., Wales D. J., Frenkel D., 2014, Phys. Rev. X, 4, 031034

Mukherjee P., Parkinson D., Liddle A. R., 2006, Astrophys. J., 638, L51

Neal R. M., 2003, Ann. Statist., 31, 705

Nielsen S. O., 2013, The Journal of Chemical Physics, 139, 124104

Pártay L. B., Bartók A. P., Csányi G., 2014, Phys. Rev. E, 89, 022302

Planck Collaboration et al., 2018, arXiv e-prints, p. arXiv:1807.06211

PÃąrtay L. B., BartÃşs A. P., CsÃąnyi G., 2010, The Journal of Physical Chemistry B, 114, 10502

Rosenbrock H. H., 1960, The Computer Journal, 3, 175

Russel P. M., Brewer B. J., Klaere S., Bouckaert R. R., 2018, Systematic Biology, 68, 219

Salomone R., South L. F., Drovandi C. C., Kroese D. P., 2018, arXiv e-prints, p. arXiv:1805.03924

Schittenhelm D., Wacker P., 2020, arXiv e-prints, p. arXiv:2005.08602

Skilling J., 2004, in Fischer R., Preuss R., Toussaint U. V., eds, American Institute of Physics Conference Series Vol. 735, American Institute of Physics Conference Series. pp 395-405, doi:10.1063/1.1835238

Skilling J., 2006, Bayesian Analysis, 1, 833

Smirnov N., 1948, Ann. Math. Statist., 19, 279

Speagle J. S., 2020, Mon. Not. Roy. Astron. Soc.,

Trotta R., Feroz F., Hobson M. P., Roszkowski L., Ruiz de Austri R., 2008, JHEP, 12, 024

Trotta R., JÃşhannesson G., Moskalenko I. V., Porter T. A., de Austri R. R., Strong A. W., 2011, Astrophys. J., 729, 106

Veitch J., et al., 2015, Phys. Rev., D91, 042003

Virtanen P., et al., 2020, Nature Methods,

\section{APPENDIX A: PROOF OF eq. (9)}

In NS we have $n=n_{\text {live }}-1$ remaining samples after the worst live point was removed. Their associated volumes were drawn from a (continuous) uniform distribution, $X_{i} \sim$ $\mathcal{U}(0,1)$. If we draw another sample, the distribution of its insertion index, $i$, relative to the other samples depends on the probability contained in the uniform distribution between the ordered samples. In fact, the probability for each insertion index $i=0,1, \ldots, n$ is

$$
\begin{aligned}
P_{i} & =\int\left(X_{i+1}-X_{i}\right) p\left(X_{i+1}, X_{i}\right) \mathrm{d} X_{i+1} \mathrm{~d} X_{i} \\
& =\int X_{i+1} p\left(X_{i+1}\right) \mathrm{d} X_{i+1}-\int X_{i} p\left(X_{i}\right) \mathrm{d} X_{i} \\
& =\left\langle X_{i+1}\right\rangle-\left\langle X_{i}\right\rangle,
\end{aligned}
$$

where we completed two trivial integrals and wrote the terms as expectations. To compute the expectations, note that

$p\left(X_{i}\right)=\frac{n !}{(i-1) !(n-i) !}\left(1-X_{i}\right)^{n-i} X_{i}^{i-1}$, 
since we need $n-i$ samples above $X_{i}, i-1$ samples below $X_{i}$ and one sample at $X_{i}$. The first factor is combinatoric; the second accounts for the $n-i$ samples that must lie above $X_{i}$; and the third accounts for the $i-1$ samples that must lie below $X_{i}$. The factor for a final sample at $X_{i}$ is just one. By integration, we quickly find $\left\langle X_{i}\right\rangle=i /(n+1)$, and thus $P_{i}=1 /(n+1)$. That is, the insertion indexes follow a discrete uniform distribution.

Note that this didn't depend especially on the fact that the distribution of the samples was uniform. If the samples had followed a different distribution, we can transform $X_{i} \rightarrow$ $Y_{i}=F\left(X_{i}\right)$ where $F$ is the cumulative distribution function, such that $Y_{i} \sim \mathcal{U}(0,1)$, the proof goes through just the same.

\section{APPENDIX B: KOLMOGOROV-SMIRNOV TEST}

We use a one-sample Kolmogorov-Smirnov (KS) test (Kolmogorov 1933; Smirnov 1948) to compare our set of $n_{\text {iter }}$ insertion indexes with a (discrete) uniform distribution. First, we compute the KS test-statistic by comparing the empirical cumulative distribution function, $F_{\text {data }}$, to that from a discrete uniform distribution, $F_{U}$,

$D_{n}=\sup _{x}\left|F_{\text {data }}(x)-F_{U}(x)\right|$.

This provides a notion of distance between the observed indexes and a uniform distribution. In the continuous case, the null-distribution of this test-statistic does not depend on the reference distribution. We convert the test-statistic into a $p$-value using an asymptotic approximation of the Kolmogorov distribution (Marsaglia et al. 2003) implemented in scipy (Virtanen et al. 2020),

$p$-value $=P\left(D_{n} \geq D_{n}^{\star} \mid H_{0}\right)$,

where $D_{n}^{\star}$ is the observed statistic. This assumes that we are testing samples from a continuous distribution. In our discrete case, the $p$-values from the Kolmogorov distribution are known to be conservative (Arnold \& Emerson 2011).

\section{APPENDIX C: TOY FUNCTIONS}

\section{C1 Gaussian}

Our first example is a multi-dimensional Gaussian likelihood,

$\mathcal{L}(\boldsymbol{\Theta})=\frac{1}{\sqrt{(2 \pi)^{n} \operatorname{det} \Sigma}} e^{-\frac{1}{2}(\boldsymbol{\Theta}-\boldsymbol{\mu})^{T} \Sigma^{-1}(\boldsymbol{\Theta}-\boldsymbol{\mu})}$,

with covariance matrix $\Sigma$ and mean $\boldsymbol{\mu}$. We pick a uniform prior from 0 to 1 for each dimension. The analytic evidence is always $\log \mathcal{Z}=0$ since the likelihood is a pdf in $\boldsymbol{\Theta}$, modulo small errors as the infinite domain is truncated by the prior. We pick $\mu=0.5$ and a diagonal covariance matrix with $\sigma=$ 0.001 for each dimension.

\section{C2 Rosenbrock}

This is a two-dimensional function exhibiting a pronounced curved degeneracy Rosenbrock (1960). The likelihood function is

$-\ln \mathcal{L}(x, y)=(1-x)^{2}+100\left(y-x^{2}\right)^{2}$.
We consider uniform priors from -5 to 5 for each parameter. The evidence can be found semi-analytically from a onedimensional integral,

$\mathcal{Z}=\frac{\sqrt{\pi}}{2000} \int_{-5}^{5}\left[\operatorname{erf}\left(10\left(5-x^{2}\right)\right)+\operatorname{erf}\left(10\left(5+x^{2}\right)\right)\right] e^{-(1-x)^{2}} \mathrm{~d} x$

to be $\log \mathcal{Z}=-5.804$. The analytic approximation, which approximates the $y$ domain of integration by the whole real line, leads to

$\mathcal{Z} \approx \frac{\pi}{20000}[\operatorname{erf}(6)-\operatorname{erf}(4)]$,

and thus $\log \mathcal{Z}=-5.763$.

\section{C3 Gaussian shells}

The multidimensional likelihood is

$\mathcal{L}(\boldsymbol{\Theta})=\operatorname{shell}(\boldsymbol{\Theta} ; \boldsymbol{c}, r, w)+\operatorname{shell}(\boldsymbol{\Theta} ;-\boldsymbol{c}, r, w)$

where the shell function is a Gaussian favouring a radial distance $r$ from the point $\boldsymbol{c}$,

$\operatorname{shell}(\boldsymbol{\Theta} ; \boldsymbol{c}, r, w)=\frac{1}{\sqrt{2 \pi} w} e^{-(|\boldsymbol{\Theta}-\boldsymbol{c}|-r)^{2} /\left(2 w^{2}\right)}$.

Thus, the highest likelihood region forms a shell of characteristic width $w$ at the surface of a $d$-sphere of radius $r$. Our likelihood contains two such shells, one at $\boldsymbol{c}$ and one at $\boldsymbol{- c}$. As usual, we take $w=0.1, r=2$ and $\boldsymbol{c}=(3.5,0, \ldots, 0)$.

With uniform priors between -6 and 6 , the analytic evidence is approximately,

$\mathcal{Z}=2\left\langle|x|^{d-1}\right\rangle S_{d} / 12^{d}$

where $S_{d}$ is the surface area of an $d$-sphere and $\left\langle|x|^{d-1}\right\rangle$ is the $(d-1)$-th non-central moment of a Gaussian, $\mathcal{N}\left(r, w^{2}\right)$, and we ignore the truncation of the domain by the finite-sized hypercube.

\section{C4 Gaussian-Log-Gamma mixture}

This toy function was found in Beaujean \& Caldwell (2013); Feroz et al. (2013); Buchner (2016) to be problematic in MultiNest without importance sampling. It is defined in even numbers of dimensions. The likelihood is a product of $d$ factors,

$\mathcal{L}(\boldsymbol{\Theta})=\prod_{i=1}^{d} \mathcal{L}_{i}\left(\theta_{i}\right)$,

where the factors are

$\mathcal{L}_{i}(\theta)= \begin{cases}\frac{1}{2} \ln \Gamma(\theta \mid 10,1,1)+\frac{1}{2} \ln \Gamma(\theta \mid-10,1,1) & i=1 \\ \frac{1}{2} \mathcal{N}(\theta \mid 10,1)+\frac{1}{2} \mathcal{N}(\theta \mid-10,1) & i=2 \\ \ln \Gamma(\theta \mid 10,1,1) & 3 \leq i \leq \frac{d+2}{2} \\ \mathcal{N}(\theta \mid 10,1) & \frac{d+2}{2}<i \leq d\end{cases}$

where e.g., $\ln \Gamma(\theta \mid 10,1,1)$ denotes a one-dimensional $\log$ Gamma density for $\theta$ with mean 10 and shape parameters 1 and 1 . There are four identical modes at $\theta_{1}= \pm 10, \theta_{2}= \pm 10$ and $\theta_{i>2}=10$.

The prior is uniform in each parameter from -30 to 30 . 
Since the likelihood is a pdf in $\boldsymbol{\Theta}$, the analytic $\log \mathcal{Z}$ is governed by the prior normalization factor, $\log \mathcal{Z}=\log \left(1 / 60^{d}\right) \approx$ $-4.1 d$, modulo small truncation errors introduced by the prior.

This paper has been typeset from a $\mathrm{T}_{\mathrm{E}} \mathrm{X} / \mathrm{LAT}_{\mathrm{E}} \mathrm{X}$ file prepared by the author. 